Recibido: julio de 2014

Aprobado: agosto de 2014

\section{LAS DINÁMICAS DE LAS ACTIVIDADES DE PRODUCCIÓN Y CONSUMO EN EL SECTOR RURAL: REFLEXIONES A PARTIR DEL CASO DEL CENTRO POBLADO (CP) DEL CORREGIMIENTO DE BERLÍN, SANTANDER*}

\author{
Karen Brigitte Vásquez**
}

DOI: http://dx.doi.org/10.15332/rev.m.v11i2.1724

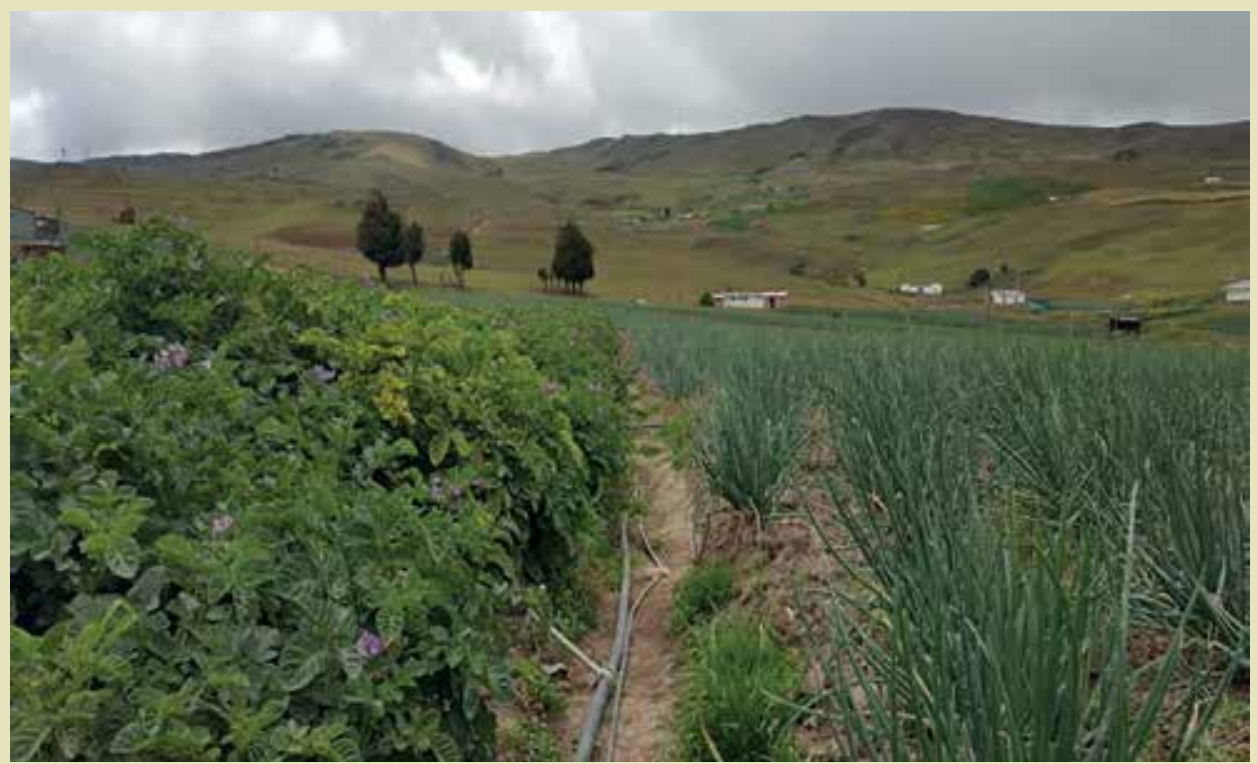

RESUMEN

La agricultura sostenible, concepto relativo a los propósitos de una nueva realidad rural, tiene lugar en un escenario natural donde grupos sociales interactúan para hallar un equilibrio deseable entre desarrollo económico y ecológico como respuesta a los Objetivos de Desarrollo Sostenible (ONU, 20I5). En este documento se presenta, al inicio, un acercamiento a la problemática rural del corregimiento de Berlín para después concentrarse específicamente en su Centro Poblado. Así bien, se exponen las connotaciones negativas que se originan en la diferencia del Estado por el sector rural para después pasar a especificidades del Centro Poblado y su área de influencia. Se abordan, por tanto, situaciones negativas provocadas por los desequilibrios relacionados con la distribución del recurso tierra, la persistencia de una agricultura ancestral, el mal manejo de agroquímicos, la inadecuada infraestructura básica de servicios, la falta de capacitación del campesino y la ausencia de servicios complementarios que propicien un desarrollo rural sostenible. Seguidamente, se identifican las debilidades y lagunas en términos de un ordenamiento territorial deseable, con base en una lectura que metodológicamente utiliza un análisis comparativo entre los instrumentos de planificación articulados hasta la fecha para el municipio de Tona y, por ende, para el corregimiento de Berlín, el cual da como resultado una metodología sugerida. En la parte final se esbozan las conclusiones más relevantes que portan a la articulación de algunas recomendaciones que se sustentan en la investigación adelantada hasta el momento (documental y de campo) y en el estudio de otros proyectos que se enmarcan en la categoría de "buenas prácticas agrícolas".

\section{PALABRAS CLAVE}

Agricultura, capital social, cultivos, desarrollo rural, ecología, externalidades, minifundios, usos, páramo, sostenibilidad. 


\section{THE DYNAMICS OF PRODUCTION AND CONSUMPTION IN RURAL AREAS: REFLECTIONS FROM THE STUDY CASE OF THE VILLAGE OF BERLÍN, SANTANDER}

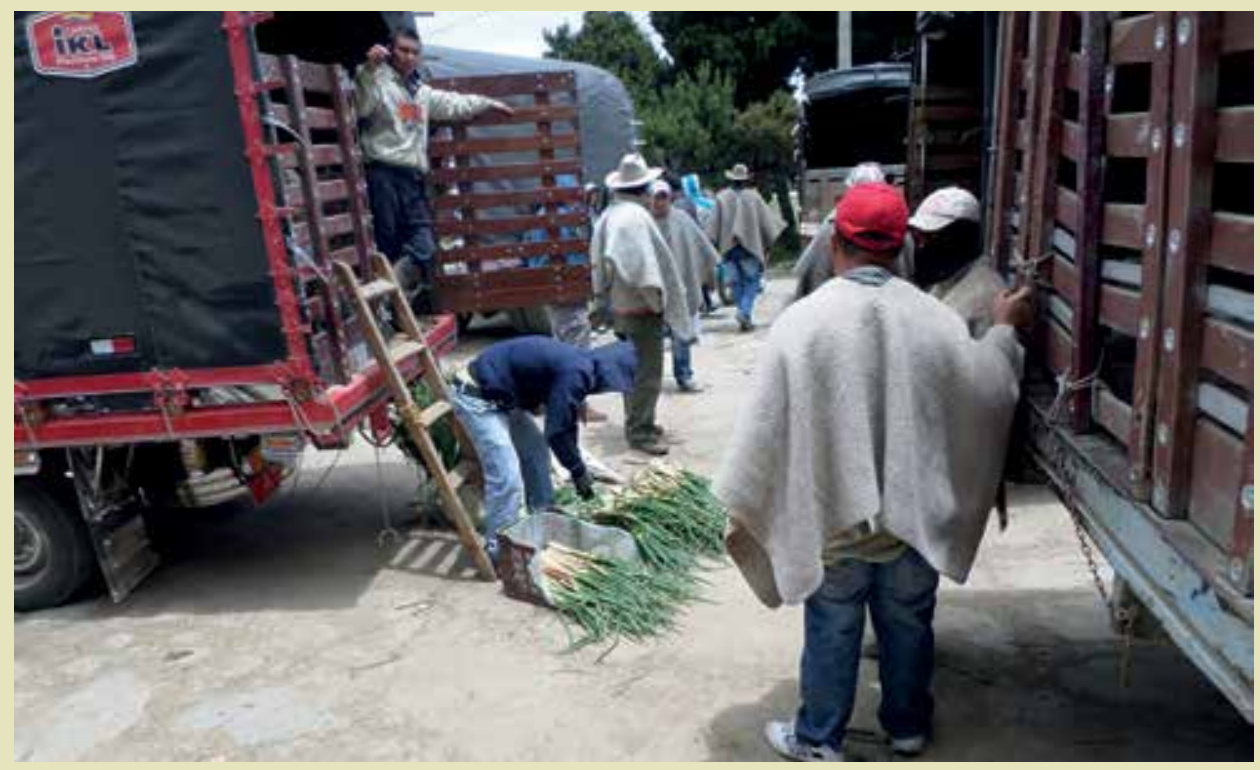

Actividades de acopio y distribución de cebolla en inmediaciones del parque del Centro Poblado del Corregimiento de Berlín Fuente: Karen Vásquez, 2014.

\begin{abstract}
Sustainable agriculture, a concept related to the purposes of a new rural reality, takes place in a natural setting where social groups interact to find a desirable balance between economic and ecological development as a response to the Sustainable Development Objectives (UN 2015). Firstly, this document presents an approach to the rural problematic of the District (corregimiento) of Berlin and then focus specifically on its urban settlement. Thus, the negative connotations that originate in the difference of the state by the rural sector are exposed, after which it is possible to move on to the specificities of the small town and its area of influence. Therefore, the paper points to negative situations caused by imbalances in land distribution, the persistence of ancestral agriculture, the poor management of agrochemicals, the inadequate infrastructure of services, the lack of training of the farmer, and the absence of complementary services that favor sustainable rural development. Next, weaknesses and gaps are identified in terms of a desirable territorial order based on a reading that methodologically uses a comparative analysis between the planning instruments articulated to date for the municipality of Tona and therefore for the Berlin area, which results in a suggested methodology. The final section outlines the most relevant conclusions that lead to the articulation of some recommendations that are based on the research carried out so far (documentary and in field) and the study of other projects that fall within the category of "best agricultural practices".
\end{abstract}

\section{KEYWORDS}

Agriculture, social capital, crops, rural development, ecology, externalities, minifundios, uses, páramo, sustainability. 


\section{INTRODUCCIÓN}

Diversos son los estudios, a veces un poco generalizadores, sobre el desarrollo rural en América Latina y cómo este territorio ha sido esculpido. Rodríguez y Meneses (20I I) hacen referencia a la ruralidad de América Latina, la cual denota significativas transformaciones en los últimos veinte años en términos de estructura productiva, dinámicas territoriales, concepción del medio ambiente y temas de gobernabilidad. De igual manera, se identifican fenómenos que sobresalen en dichos ámbitos:

a. La importancia que cobra cada día la economía rural que se basa en otras actividades diferentes a la agricultura, sin que esto demerite la importancia estratégica de esta actividad.

b. La imperiosa necesidad de superar la dicotomía existente entre lo urbano y lo rural, ya entendida por muchos grupos sociales.

c. La preocupación, cada día más, por el aumento del deterioro de los recursos naturales y todos los impactos que genera el cambio climático.

d. Las mutaciones de las instituciones rurales, especialmente en términos peyorativos que tienen que ver con una pronunciada debilidad de los mecanismos tradicionales usados por el Estado para intervenir en el territorio rural, así como la intervención de nuevos esquemas que propenden por una participación mayor y más activa de los actores locales.

En síntesis, una serie de razonamientos esbozados como oportunidades aprovechables en la búsqueda de un desarrollo rural más sostenible e inclusivo. A pesar de esto, las metodologías referidas al desarrollo rural en Colombia han sido durante muchos años ambiguas e inconsistentes, por lo que el crecimiento y desarrollo de la población así como la productividad del suelo se han visto rezagados, dictamen que se refleja en una serie de indicadores de calidad de vida, de oportunidades laborales e índices de ingresos que individualmente $o$ en su conjunto no dejan de ser preocupantes. Lamentablemente, se pasan por alto las grandes potencialidades del territorio rural colombiano, las cuales van desde el plano socioeconómico como el turismo ecológico y el paisajismo hasta los de neta índole geográfica como fertilidad del suelo, clima, topografía, recursos hídricos para seguir cayendo en la adopción repetitiva de una agricultura convencional caracterizada por las malas prácticas, los monocultivos y un notable desequilibrio ecológico. Se llega entonces al corregimiento de Berlín, territorio por analizar en la investigación que fundamenta este artículo y que se ubica en suelos de gran importancia ecosistémica con potencial para un consistente proyecto de desarrollo rural sostenible. El corregimiento de Berlín pertenece al municipio de Tona que se localiza al nororiente de la cuenca superior del río Lebrija, en la Provincia de Soto, departamento de Santander (figura I).

A su vez, el municipio de Tona se subdivide en 16 veredas situadas en diferentes pisos térmicos. El Centro Poblado del corregimiento de Berlín se posiciona dentro de los límites del páramo Santurbán, clasificado como Distrito de Manejo Integrado (DMI) de Berlín, en la convergencia de las veredas de Ucatá, Saladito y Cuestaboba, exactamente sobre la vía nacional que conecta Bucaramanga y Cúcuta (figura 2). 

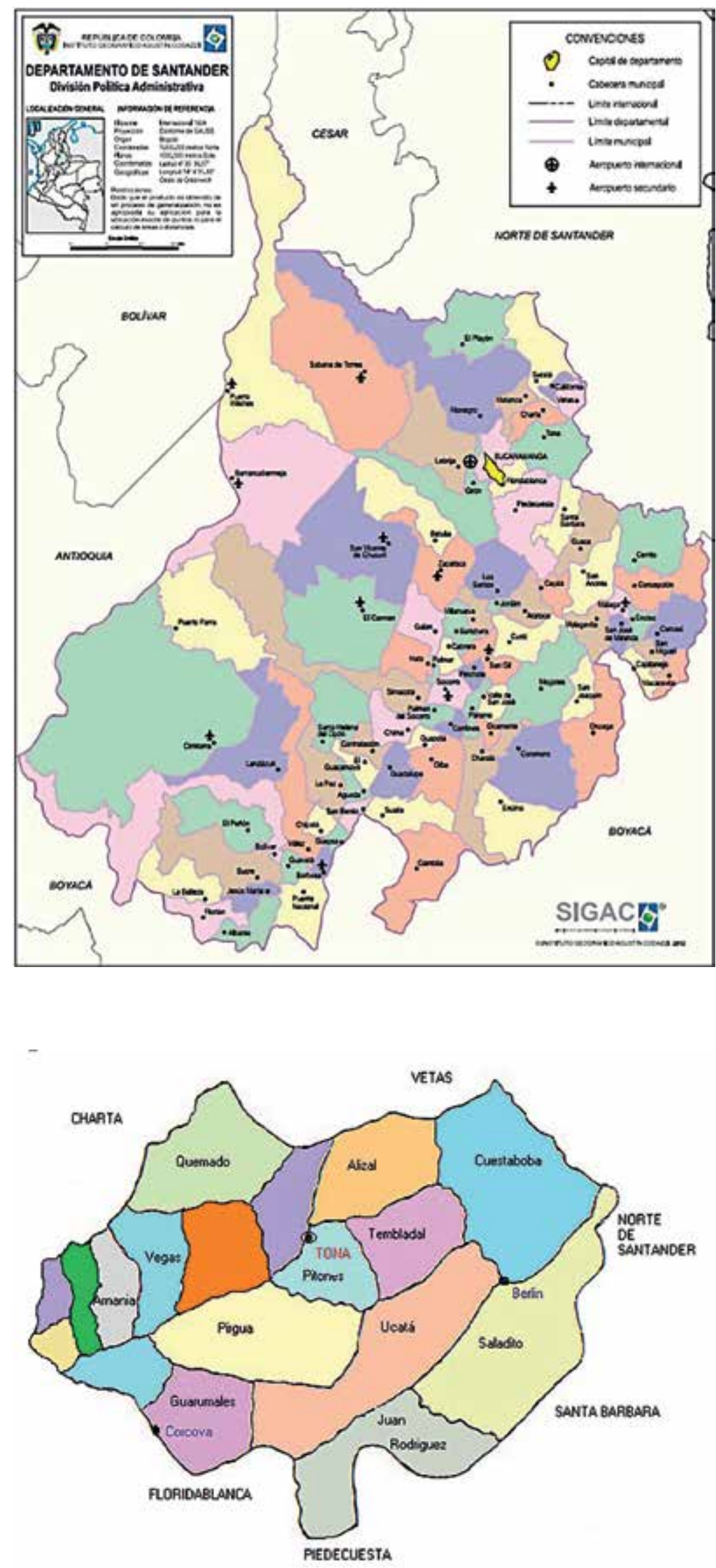

Figura I. Ubicación del municipio de Tona, en relación al departamento de Santander Fuente: www.igac.gov.co
Figura 2. Subdivisión del municipio de Tona en 16 Veredas

Fuente: $h t t p: / / w w w . t o n a-s a n t a n d e r . g o v . c o /$ 
El páramo seco de Berlín que hace parte del Parque Nacional Santurbán es atravesado por una vía nacional que conecta las ciudades de Bucaramanga y Cúcuta. Allí, sobre ambas márgenes del asfalto se asienta el Centro Poblado del Corregimiento de Berlín, uno de los dos corregimientos del municipio de Tona, sobre un escenario natural agreste y sugestivo que cada día se ve agobiado por malas prácticas originadas por una antropización depredadora y en el letargo social y económico de la población residente. En este orden de ideas, se busca con la investigación, in situ y documental, identificar los componentes relativos al "subsistema de actividades de producción y consumo" (Gómez, 2008), a fin de establecer elementos de juicio para proponer una adecuada metodología que contribuya a identificar ordenadamente los problemas atinentes al mal manejo del suelo para posteriormente equilibrar, reformular o proponer ciertas políticas de ordenamiento territorial. El artículo finaliza entonces con un aparte donde se vincula a la comunidad como eje central de los lineamientos del Estado y se le posiciona como "actor protagónico", cuyo rol es velar por un adecuado uso del suelo y de los recursos naturales presentes en el territorio estudiado. En consecuencia, el cambio estructural planteado se caracteriza sustancialmente por el tránsito de una economía rural dominada por una agricultura convencional, causante de un alto grado de contaminación ambiental, a una economía rural más ecológica y diversificada, con actividades complementarias de mayor valor agregado (vinculadas o no a la agricultura), naturalmente con una creciente participación de esta comunidad que hasta el momento no goza de beneficios y oportunidades cimentadas en el concepto de equidad.

\section{LAS PROBLEMÁTICAS EN EL CORREGIMIENTO DE BERLÍN COMO PUNTO INICIAL DE REFLEXIÓN}

La agricultura ha desempeñado un papel decisivo en el crecimiento de la mayoría de las economías de América Latina y Colombia no es una excepción. Sin embargo, el país presencia desde hace varias décadas una fuerte crisis del sector agrícola, lo que representa un obstáculo para el desarrollo territorial. En el caso del corregimiento de Berlín muchos problemas son concomitantes a los de América Latina, aunque buena parte obedecen a circunstancias particulares provocadas por circunstancias sociopolíticas propias del país. Por tal motivo, el análisis de la agricultura exige profundizar, en los problemas inherentes al uso del suelo identificados en el área estudiada, los cuales se han agudizado por el olvido del Estado hacia el sector rural y los problemas de orden público que allí se han gestado.

A esto se suman también las dinámicas endógenas presentes en función de la propiedad de la tierra, la situación de los productores y las hileras de distribución que regulan la rentabilidad y el destino de la producción agrícola. La distinción canónica entre minifundios y latifundios en las zonas agrícolas es insuficiente para entender el calidoscopio de la tenencia de la tierra, que en otras palabras se traduce en un acentuado desequilibrio de las áreas de producción pertenecientes en su mayoría a terratenientes y en una notable posición de desventaja a los campesinos. A estos regímenes de la tenencia de la tierra se suman situaciones que afronta el campesino en cuestiones de contratación que se pueden sintetizar en salariados, semi-salariados o trabajadores fijos o de temporada que se amalgaman con la mano de obra familiar y múltiples formas "arcaicas" de trabajo comunal de ayuda mutua. Oxfam (2016) conduce estudios al respecto que también ahondan en los diversos tipos de agricultura de subsistencia que aportan pequeñas cuotas en el mercado de la producción, pero que en cualquier caso no elevan el tenor de vida de la población rural. De igual manera, se hace referencia a problemas de una agricultura que no se integra adecuadamente a la industria y al comercio por indiferencia de la administración pública y una arraigada idiosincrasia de la población. En síntesis, una baja productividad como principal problema no solo del 
corregimiento de Berlín sino de la agricultura de muchos países de América Latina, la cual impide liberar el excedente suficiente para importar bienes industriales y herramientas para direccionar la economía (Oxfam, 2016).

En este orden de ideas, la ausencia de políticas coordinadas para que los beneficios procedentes de la producción se reviertan, en parte, en el territorio de origen es evidente. Sin duda, un nudo en la cadena de problemas que se une a aspectos que requieren un profundo estudio y que a simple vista también aquejan al páramo de Berlín. Al concentrar las observaciones realizadas en torno al Centro Poblado del corregimiento de Berlín y a la búsqueda de información durante la investigación documental surgieron vacíos por la falta de estudios especializados e inclusive del hermetismo de la población. En cualquier caso, es más que evidente que la tierra en el Centro Poblado clama por soluciones a problemáticas como los territorios baldíos y la desigualdad en la tenencia de la tierra, donde es claro un usufructo del campesino por parte del empresario que ve el territorio como fuente de rentabilidad, en oposición a la visión del campesino quien lo considera como patrimonio y bien heredable para hacer su vida en el campo. En otras palabras, una brecha de desigualdad para acceder a la propiedad de la tierra, dimensión prácticamente desconocida en ausencia de un censo para determinar quiénes son los dueños de la tierra, así como de un fondo de tierras o leyes de concertación.

Por fortuna, el Centro Poblado de Berlín por su posición estratégica no se encuentra afectado directamente por situaciones exógenas relacionadas con los índices de violencia en el sector rural colombiano, según lo declarado por la Policía Nacional presente en el Centro Poblado. En cualquier caso, sí debe lidiar con problemas endógenos como disputas activas por la tenencia de la tierra, una tensa convivencia por sus intereses e inclusive situaciones puntuales en las que se presentan amenazas, asesinatos o amedrentamientos.

Según entrevistas no estructuradas que se realizaron a algunas personas, casi siempre la gran propiedad se estableció gracias a la usurpación de las tierras, la expropiación de campesinos arruinados o muy endeudados, y la apropiación de tierras públicas por privados con la ayuda o el consentimiento del Estado. Asimismo, la ignorancia y la falta de información han provocado revueltas campesinas que se deben generalmente a la penetración del capitalismo en el campo y por la negativa de un proceso de modernización que priva a los agricultores de los derechos adquiridos. Entran aquí en juego todas las dinámicas del llamado "Plan Integral de Manejo del Distrito de Manejo Integrado de los Recursos Naturales Páramo de Berlín", comúnmente denominado como DMI, en práctica desconocido o mal interpretado por el campesino, lo que dificulta una clara concertación con operadores territoriales capacitados para cuestiones relacionadas con el desarrollo rural, entre estos el mejoramiento de la productividad del suelo.

Específicamente, las zonas aledañas al Centro Poblado esbozan un paisaje agrícola que corrobora la intervención generalizada del hombre en los páramos en las últimas décadas y que, en este caso, se basan en actividades del sector primario en cultivos de papa y cebolla, por ser especies vegetales que se adaptan fácilmente a las inclementes condiciones climáticas del territorio. Sin embargo, este uso del suelo ha ocasionado el drenaje e incluso la desecación de fuentes hídricas, así como la quema de frailejones y otras especies de plantas propias de los páramos. Paralelamente, campesinos de los sectores afectados han advertido la notable disminución de los caudales en las quebradas, que en muchos casos se han secado debido a que sus nacimientos han cesado de emanar agua. En razón de estos hechos, durante los años 1997 y 2000 se desarrollaron investigaciones que permitieron 
conocer el impacto causado a los páramos por la implantación de cultivos de papa y otras actividades como la ganadería (Avellaneda-Cusaría, 1997-1999). Posteriormente, el ICA y otras instituciones $(20 \mathrm{II}$ ) emiten una serie de documentos técnicos pertenecientes a una denominada "Línea Agrícola" que se dedica a estos aspectos. En libro Manejo fitosanitario del cultivo de papa (Solanum tuberosum, subsp. andigena y S. phureja) se compendian los principales problemas fitosanitarios que afectan el cultivo de papa: el gusano blanco (Premnotrypres vorax), la polilla guatemalteca (Tecia solanivora), palomilla (Phtorimae operculella) y la gota (Phytophthora infestans), lo cual genera pérdidas económicas y conlleva la masiva utilización de plaguicidas para el control de estas plagas. El cultivo de cebolla larga también tiene problemas fitosanitarios como: minador de la cebolla (Liriomyza huidobrensis), babosas y caracoles (Deroceras reticulatum Müller), babosa pequeña (Limax marginatus Müller), babosa parada rayad (Milax gagates draparnaut), babosa gris (Helix aspersa), Trozadores o tierreros (Agrotis ipsilon Hufnagel), entre otras. Otra problemática en este cultivo es el uso inadecuado y sin procesar de la pollinaza o gallinaza, por ejemplo, el efecto negativo que tiene almacenarla durante poco tiempo con bajo nivel de nitrógeno, siendo más difícil la descomposición de la materia orgánica y la renovación de los componentes del suelo.

Según Monroy (2009), las transformaciones que afectan los ecosistemas naturales en zonas de páramo para dar paso a un sistema agrícola (descapote, arado, abonos, etc.) provocan notables alteraciones de orden físico sobre el suelo que se revierten en la desaparición de la protección física de este y generan una mayor susceptibilidad a ataques microbianos o en su defecto una mineralización de la materia orgánica. De igual forma, Monroy (2009) indica que la susceptibilidad de las poblaciones microbianas disminuyen vertiginosamente en el momento en que la vegetación natural se ve alterada, es por ello que el uso intensivo del suelo del páramo genera riegos de orden ambiental que se manifiestan en la acumulación de $\mathrm{CO}_{2}$, problemas de fertilidad de los suelos, la capacidad hidráulica y la misma estabilidad del suelo.

Regresando nuevamente, los problemas del asentamiento urbano, o sea el Centro Poblado como tal, es notable que el tejido urbano aqueja problemáticas relacionadas con el espacio público y privado: comercio formal e informal se amalgaman con el uso residencial en viviendas que no están servidas completamente por una adecuada infraestructura básica de servicios. No fue difícil identificar la presencia de un incipiente sistema de acueducto y el déficit de un sistema de alcantarillado que afecta directamente los recursos hídricos de la zona y en consecuencia la producción agrícola como se expuso precedentemente en los problemas fitosanitarios.

Asimismo, el Centro Poblado experimenta problemas de un crecimiento urbano desordenado y poco planificado, que inicia en los lotes baldíos que gravitan alrededor del parque central y se extiende a sus zonas periurbanas. El parque central ha perdido su carácter original ante su abandono y descuido, pocas personas lo frecuentan, ya que su mobiliario urbano está completamente deteriorado. Solo una cancha de cemento reactiva el área en algunos momentos de una monótona semana, que solo espera la llegada de los sábados y domingos para los intercambios comerciales que se concentran en los negocios situados sobre los costados del parque y que a su vez sirven como centro de acopio de los productos agrícolas de la zona. Por su parte, el comercio informal situado sobre el eje vial entre Bucaramanga y Cúcuta es el primer beneficiario de una actividad comercial no planificada y que invade los retrocesos de norma previstos para el perfil vial actual que, en cualquier caso, es obsoleto y requiere de una modernización urgente (figura 3). 

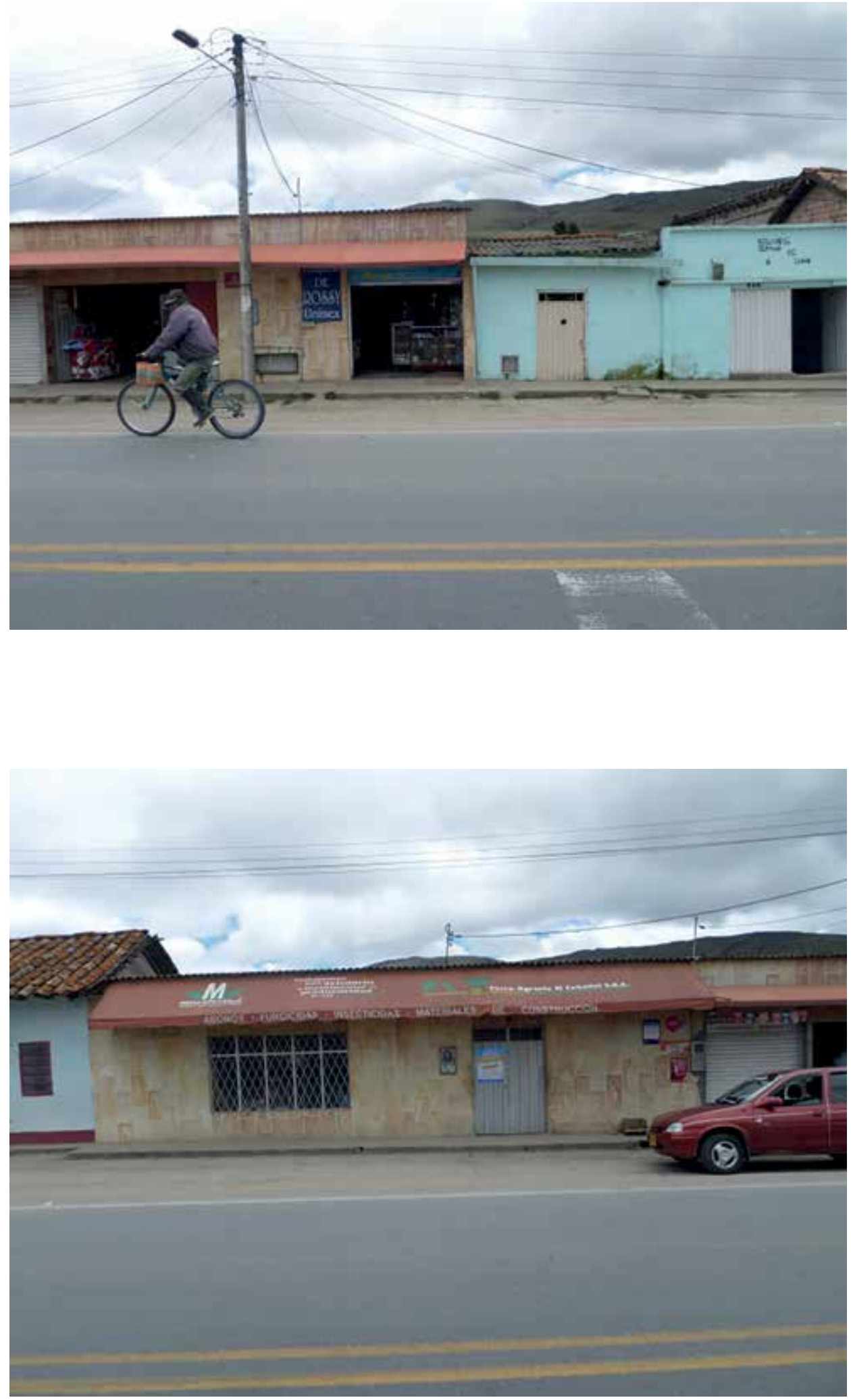

Figura 3. Dos aspectos de la vía nacional que conecta Cúcuta y Bucaramanga Fuente: Karen Vásquez, 2014

M 53 REVISTA M VOL. 11 No.2. JULIO-DICIEMBRE 2014 • FACULTAD DE ARQUITECTURA • UNIIERSIDAD SANTO TOMÁs COLOMBIA 


\section{LA CONFRONTACIÓN DE LA REALIDAD PRODUCTIVA DEL CENTRO POBLADO DEL CORREGIMIENTO DE BERLÍN CON LOS INSTRUMENTOS DE PLANIFICACIÓN DISPONIBLES Y LA SITUACIÓN DESEABLE PLANTEADA POR LA FAO}

La agricultura, como cualquier actividad humana, posee una serie de matices gracias a una evolución histórica. Por tanto, esta labor dejó de catalogarse como una actividad de autoconsumo al evolucionar hacia nuevas dinámicas socio-económicas de producción, en su mayoría reguladas por una normativa específica relacionada con la producción. Posterior a la producción están los excedentes comercializables, ya sea por trueque o por las habituales transacciones monetarias. A medida que la agricultura evolucionó también lo hicieron los métodos y los tipos de cultivos, aunque no para bien en muchos casos. En efecto, la FAO (2005) denuncia en sus reportes cómo las prácticas agrícolas, nuevas o tradicionales, utilizan una gran cantidad de insumos químicos que han dado lugar a un desequilibrio en la fertilidad del suelo, la pérdida de la biodiversidad e inclusive la disminución de las cosechas. En virtud de los resultados arrojados por estas nocivas prácticas agrícolas. En 1992, durante la Conferencia de las Naciones Unidas sobre el Medio Ambiente y el Desarrollo (conocida como Cumbre de la Tierra) se crea un documento de 40 capítulos, organizados en un preámbulo y cuatro secciones, de los cuales el número 14 imparte las directrices para el fomento de la de agricultura y desarrollo rural sostenible (ADRS). Así bien, se inician a consolidar "buenas prácticas" en la agricultura y además a reforzar las instituciones para que apliquen estos modelos en varios países del mundo bajo la supervisión y guía de la FAO (I).

En este orden de ideas, la investigación confronta desde el reconocimiento del territorio del Centro Poblado la situación actual de la agricultura circunscrita en sus alrededores y el estado de los asentamientos allí presentes con las directrices de la FAO, lo planteado en los instrumentos de planificación territorial del municipio y los niveles de intervención del Estado en la zona; este último aspecto fundamentado, en parte, por un reconocimiento visual directo y en indicadores desactualizados. Al respecto, cabe anotar que durante el proceso de investigación se buscó documentación oficial histórica que registrase la inclusión de la agricultura rural sostenible en los procesos de planificación sin ningún resultado satisfactorio.

Así las cosas, el análisis se registra en la tabla I, estructurada con base en los instrumentos de planificación encontrados y con referencia a cuestiones del subsistema de producción y consumo. Estos son: Esquema Básico de Ordenamiento Territorial (EOT) del 2000, Plan de Desarrollo Municipal 2016-2019 y el Distrito Manejo Integrado (DMI) 2009-20I9.

\section{La formulación de un instrumento para el correcto análisis del subsistema de producción y consumo en el Centro Poblado del corregimiento de Berlín}

La correcta lectura del territorio es una de las principales exigencias para poder proponer un acertado modelo metodológico para el reconocimiento de los subsistemas del territorio, en este caso, el subsistema de producción y consumo en inmediaciones del Centro Poblado de Berlín. Es así que para el correcto análisis del subsistema se consolidó una lista de características que a su vez generan indicadores cualitativos y cuantitativos que permiten organizar y legitimar la investigación. Además, la definición de las características son un punto de partida para profundizar en los problemas, enfrentar, articular el diagnóstico y crear una metodología que lleve a una solución compatible con las necesidades y las características del medio natural y la población. 
Tabla I. Cuadro comparativo de los contenidos de los instrumentos de planificación y gestión del municipio de Tona en relación con la situación actual del Centro Poblado (CP) del corregimiento de Berlín

\begin{tabular}{|c|c|c|c|}
\hline INSTRUMENTO & $\begin{array}{l}\text { Esquema Básico de } \\
\text { Ordenamiento } \\
\text { Territorial }\end{array}$ & $\begin{array}{c}\text { Plan de Desarrollo } \\
\text { Municipal }\end{array}$ & $\begin{array}{l}\text { Plan Integral de } \\
\text { Manejo del Distrito de } \\
\text { Manejo Integrado de } \\
\text { los Recursos Naturales } \\
\text { "páramo de Berlín" }\end{array}$ \\
\hline \multicolumn{4}{|l|}{ ITEM } \\
\hline $\begin{array}{l}\text { Caracterización y zoni- } \\
\text { ficación de sistemas de } \\
\text { producción }\end{array}$ & $\begin{array}{l}\text { En el EOT se busca crear } \\
\text { cartografía del municipio } \\
\text { según la actividad econó- } \\
\text { mica por sectores (prima- } \\
\text { rio, secundario y terciario) } \\
\text { en zona rural y urbana. }\end{array}$ & $\begin{array}{l}\text { No ofrece información } \\
\text { sobre cartografía de ac- } \\
\text { tividades económicas. } \\
\text { Solamente se registra un } \\
\text { inventario de cultivos, } \\
\text { cría de animales, estable- } \\
\text { cimientos comerciales y } \\
\text { minería (mármol y caliza } \\
\text { ubicada en el corregimien- } \\
\text { to de Berlín). }\end{array}$ & $\begin{array}{l}\text { En el capítulo } 3 \text { del docu- } \\
\text { mento, titulado "Ordena- } \\
\text { miento territorial y zoni- } \\
\text { ficación", se tiene la car- } \\
\text { tografía y el proyecto de } \\
\text { actualización de las zonas } \\
\text { destinadas a la producción } \\
\text { urbana y rural, a las zonas } \\
\text { de recuperación para la } \\
\text { producción. Se especifica } \\
\text { para la primera zona, el } \\
\text { área del corregimiento de } \\
\text { Berlín con } 37 \text { ha. Para la } \\
\text { segunda zona se habla del } \\
\text { corredor comprendido } \\
\text { entre el corregimiento } \\
\text { de Berlín y Silos, con usos } \\
\text { compatibles para la agri- } \\
\text { cultura orgánica y ecoló- } \\
\text { gica, teniendo como uso } \\
\text { prohibido la agricultura } \\
\text { intensiva. }\end{array}$ \\
\hline
\end{tabular}

EI EOT tiene como base la información desactualizada de 1993, suministrada por la Corporación Autónoma Regional para la Defensa de la Meseta de Bucaramanga (CDMB), en su programa agropecuario municipal. El corregimien-

Inventario de tenencia de tierra y tamaño predial

Índices Producción agrícola to de Berlín conformado por las verdeas de Ucata, Cuestaboba y El Saladito, registran en términos de tenencia de tierra propia: Ucata 125 predios propios y 84 aparceros, Cuestaboba 61 predios y 50 aparceros, El Saladito 94 predios propios y 50 aparceros.

En este documento se abordan los cultivos principales de esta área del corregimiento de Berlín. Entre ellos se encuentra el cultivo transitorio de papa con un rendimiento de $10.000 \mathrm{~kg} / \mathrm{ha}$, área cultivada de 700 ha, posicionándose como cuarto productor en el departa-
El documento no ofrece información sobre tenencia de tierra y tamaño predial de la zona del corregimiento de Berlín.
El documento no se pronuncia específicamente sobre tenencia de tierra y tamaño predial. mento de Santander; el cultivo semi-permanente de cebolla larga con rendimientos de $20000 \mathrm{~kg} / \mathrm{ha}$ y con un promedio de 440 ha cultivadas; el pasto de corte 6886 ha y el trébol y kikuyo 6638 ha (datos del año 1993).

Este instrumento presenta datos del 2015, teniendo diferencias en los rendimientos de los cultivos: la papa se clasifica como cultivo anual y con rendimientos de $50 \mathrm{tn} / \mathrm{ha}$; cultivos semi-permanentes de cebolla larga con rendimientos de 28,8 tn/ ha y con un promedio de 1500 ha cultivadas, el pasto de corte 500 ha, kikuyo I 2.000 ha. No hay información sobre área cultivada de trébol.

\author{
Los datos en este docu- \\ mento son del año 2007, \\ no se clasifican los culti- \\ vos según su duración y \\ se tiene para la zona del \\ corregimiento de Berlín \\ el cultivo de cebolla larga \\ 610 ha, papa 870 ha y en \\ pastos 33 ha.
}




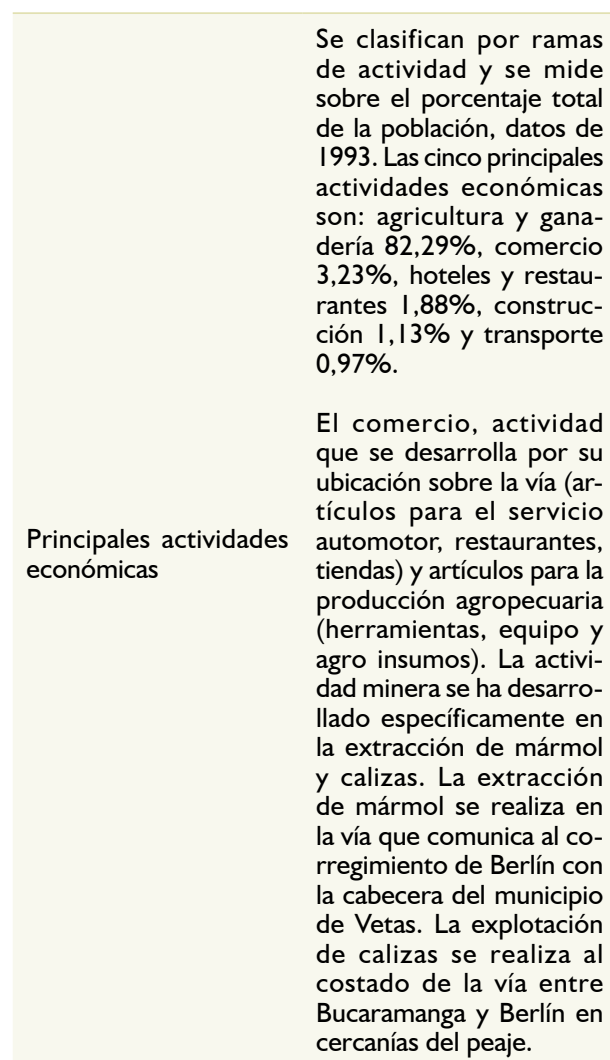

La producción pecuaria no se lleva a cabo con intensidad en el área del corregimiento de Berlín.

Índices de producción pecuaria
El documento habla de actividades económicas en las zonas urbanas, esto incluye el centro poblado de Berlín, pero no lo disgregan. Los tres principales establecimientos que tienen influencia en e PIB municipal son: comercio con $57,7 \%$, servicios $29,6 \%$ e industria con el $12,8 \%$.
El documento no presenta información acerca de las actividades económicas.
El análisis que se hace es a nivel de municipio y la única información en relación con el corregimiento de Berlín en la cría de animales para autoconsumo, pero no se especifican variedades o razas, ni cantidades.
Se hace una pequeña referencia en el capítulo 2 de actividades económicas pecuarias ubicadas en las veredas El Saladito y Cuesta Boba correspondientes al corregimiento de Berlín, en las que se destaca la cría de ganado y ovejas, a escala familiar tenemos la avicultura y piscicultura.

En el capítulo 5 se exponen los proyectos sobre desarrollo sostenible, entre ellos:

Conocimiento, conservación y uso sostenible de la biodiversidad en el páramo de Berlín. Desarrollo de proyectos productivos sostenibles y competitivos en las zonas de producción y de recuperación para la producción. Planeación y fomento del ecoturismo como alternativa sostenible de generación de ingresos dentro de la estructura de cadena pro-

ductiva en el área del DMI y el fondo agropecuario de garantías para adquirir nuevas tecnologías y mejores procesos.

En el capítulo de proyecnos compatibles con e de las UMATA, capacitatas etnomedicinal propias de leyes y conocimiento de la clasificación de tieaprovechamiento de agricultura sostenible, dinamización de los instrumentos financieros como el incentivo de capitalización rural
No existen posib máticas. 
Tabla 2. Ficha de características y elementos del subsistema de producción y consumo

\begin{tabular}{ll}
\hline Elementos del subsistema & Características por determinar de cada subsistema \\
\hline Actividades de producción & Sector Primario: Actividades agropecuarias \\
Actividades de consumo & Sector Secundario: Industria \\
Sector Terciario: Servicios \\
Capital social \\
Población ocupada según sector económico \\
Ingreso per cápita del corregimiento de Berlín y del Centro habitado. \\
Capital humano, innovación y conocimiento \\
Externalidades positivas y negativas \\
Cartografía actualizada de predios. \\
Tipología de los cultivos (2) \\
Zonas de protección y zonas de erosión \\
Cartografía de fuentes hídricas \\
Censo actualizado \\
PNB del corregimiento vs municipio \\
Tipología de trayectorias \\
NBI (Necesidades básicas insatisfechas) \\
Cambios de parámetros económicos \\
Informe de sistema de producción y tipos de cultivos \\
Análisis de sistema de riego
\end{tabular}

Fuente: Karen Vasquez, 2014

\section{¿Cuáles buenas prácticas para el CP de Berlín?}

Si bien, las líneas guías generales, formuladas por investigadores y planificadores, para sentar las bases de un desarrollo rural sostenible son útiles como referencia en las diferentes fases que conlleva el estudio de un territorio, es también cierto que cada lugar establece sus particularidades y exigencias físicas, sociales y económicas (Gómez, 2007). Por tanto, buscar una lógica línea de intervención depende de la necesidad de adaptar o enfocar las políticas de gestión de recursos naturales y el respeto de las características morfológicas de la tierra hacia el mejoramiento de la seguridad alimentaria. De la misma forma, el desarrollo según las necesidades de la población en el intercambio de bienes y servicios, propone dos figuras importantes como respuesta a la problemática: una es el ordenamiento territorial de cada municipio, el cual asigna los usos adecuados a las capacidades del territorio en cuestión (Gómez, 2007). La otra es la planificación particularizada de las medidas de desarrollo rural; las cuales al confluir se complementan, y generan un ambiente propicio para que se dé el llamado desarrollo sostenible. En este orden de ideas (González, 2009) afirma que en "la búsqueda de instrumentos de gestión que posibiliten la transversalidad de políticas sectoriales, diversos países han encontrado en el manejo integral de cuencas un instrumento de planeación y de gestión adecuado" (3).

Por su parte, Gómez (20l3) establece que el ordenamiento territorial rural sostenible debe responder a los siguientes objetivos:

I. Frenar las degradaciones ecológicas y despilfarro de recursos naturales: evitar la incorrecta selección de actividades soportadas en un territorio.

2. Considerar los riesgos naturales en la localización de actividades.

3. Evitar una mezcla de superposición desordenada de uso.

4. Reducir los problemas derivados del desequilibrio territorial: evitar la congestión de unas zonas y la desertificación de otras. 
5. Mejorar la accesibilidad, tanto de recursos como a lugares de trabajo. Considerar las dificultades territoriales para dotar de equipamientos y servicios a la población.

6. Superar los conflictos entra actividades y sectores.

7. Facilitar la coordinación entre organismos públicos.

En el panorama latinoamericano, algunos proyectos viables pilotos apropiados en otras regiones de similares características como en Camancha (Chile), apoyados por la FAO, han logrado éxito gracias al uso del sistema de captaniebla, el cual consiste en atrapar las gotas microscópicas de agua en la niebla por medio de una malla plástica y de construcción con elementos reciclables. Otro proyecto es el turismo científico social, apropiado en el desierto de Atacama (Chile), por su fragilidad y al ser una región única en el mundo se presenta como ventaja comparativa frente al turismo científico social. Asimismo, merecen atención otros proyectos en los cuales se da gran importancia a la capacitación y al conocimiento de otros cultivos aptos para su suelo, como la quinua, la cebada y el trigo, así como la consolidación de un ente asesor, capacitador y de control para las "buenas prácticas agrícolas".

\section{CONCLUSIONES Y RECOMENDACIONES}

El reconocimiento del Centro Poblado del corregimiento de Berlín funge como elemento cardinal para identificar las dinámicas y características de la producción agrícola y las formas de comercio (consumo) presentes en el núcleo urbano y su entorno, así como las principales problemáticas que lo aquejan e impiden un desarrollo sostenible de este territorio. Estas se pueden resumir puntualmente de la siguiente manera:

\section{En términos de producción}

- Gran parte de la población visualizada ubicada en la periferia cuenta con forma de propiedad en minifundio con cultivos de papa (solanum tuberosum) y ajo (allium fistulosum) cebolla larga.

- Poseen cría de animales de granja para autoconsumo pero no ven la pecuaria como una actividad que bien puede generar otros ingresos.

- Su método de producción agrícola es convencional, o sea, basado en la agroquímica.

- La producción agrícola se divide en dos aspectos: el autoconsumo por pequeños excedentes y los cultivos no diversificados con destinación comercial.

- Semillas autóctonas, con modificaciones propias por clima e injerto con otro tipo de semillas, no tienen registro en el Instituto Colombiano Agropecuario (ICA).

- Se evidencia explotación del ager (territorio cultivado) bajo el sistema de monocultivo con cultivos transitorios y semipermanentes; no hay dinámica de rotación con otros cultivos aptos para suelos secos y de páramo (3).

- Hay una evidente problemática con externalidades negativas a causa del uso indebido de agroquímicos y mal manejo de fuentes hídricas, así como la falta de interés del Estado por proyectos de ADRS y BPA. 
- No se visualiza capital social entre los agentes principales del subsistema de producción y consumo, esto tiene como consecuencia la falta de infraestructura de acopio agrícola.

- En el Centro Poblado aparecen en su periferia áreas considerables de cultivos periurbanos, los cuales se desarrollan como una fuente básica de sostenimiento económico, sin pensar en nuevos roles para asignar a la agricultura (Gómez, 2009). Una pequeña excepción se evidenció en los programas de capacitación e innovación agrícola del colegio del núcleo urbano.

- El sector terciario es estimulado principalmente por la vía nacional entre el municipio de Bucaramanga (Santander) y Pamplona (Norte de Santander).

- Se evidencia comercio formal e informal, principalmente en el sector alimenticio.

\section{En términos sociopolíticos}

La sociedad residente en el Centro Poblado afronta una serie de problemas que parten desde la imposibilidad del campesino para acceder a la propiedad de la tierra. Es un hecho que existe una disputa activa por la tenencia de la tierra, en la cual la mejor partida la llevan los terratenientes empresarios que solo ven el territorio como un escenario netamente de producción y rentabilidad económica, en contraste con la visión del campesino quien ve la tierra como un patrimonio heredable sobre el cual construir un modelo de vida. Por tanto, el Centro Poblado mira impotente una realidad incierta en términos de desarrollo socioeconómico, el cual se caracteriza actualmente por la obsolescencia, la inequidad y un marcado desbalance en el régimen de activos y pasivos territoriales.

A esta situación se agregan los índices de violencia que agobian el páramo, imperceptibles a primera vista, pero que están allí latentes, debido a un fallido estado ideal caracterizado por una convivencia pacífica por los intereses de cada uno de los campesinos. No es un secreto que la lucha por la tierra y el trabajo genera amenazas, amedrentamientos e inclusive asesinatos, en muchas ocasiones sustentados por secuelas del conflicto armado, cuyo cese actualmente propiciado por el Gobierno Nacional es insuficiente y no cubre muchos aspectos.

Paralelamente, se presentan problemas originados en la distribución de recursos para el usufructo del suelo y en la corrupción dentro de los ambientes de las hileras de distribución. Así las cosas, con la reforma tributaria planteada por el Estado los problemas se acentúan, pues en cambio de soluciones siguen apareciendo fenómenos contradictorios. La otra cara de la moneda es el discutible manejo de las medidas redistributivas de la tierra, aspecto que acusa grandes vacíos e interrogantes ante la usencia de un censo oficial que identifique quiénes son los dueños de la tierra. Según conversaciones con los campesinos, muchos han perdido sus parcelas por problemas económicos y estratagemas económicas de los terratenientes que en poco caso son dirimidas por inconsistentes procesos judiciales.

\section{En términos de planificación territorial}

Según lo identificado en la tabla I es evidente que la administración pública ha descuidado un buen número de componentes relativos a la planificación y gestión del territorio. Las razones pueden ser múltiples, pero las principales soslayan en la baja capacitación de los funcionarios públicos sobre estos temas, la escasa asignación de recursos presupuestales e inclusive una marcada indiferencia por parte de la administración. Lo anterior conduce a 
un incipiente número de instrumentos de planificación basados en indicadores fidedignos y actualizados, como lo son un censo predial, una ley de concertación y un eventual fondo de tierras, visto que hay buena cantidad de predios sin un dueño aparente. En síntesis, no existe un Plan de Desarrollo Rural particularizado para el Centro Poblado del corregimiento de Berlín que atienda las exigencias endógenas de este territorio. Esta aseveración se fundamenta una vez más en el proceso de confrontación de los actuales instrumentos de planificación territorial (tabla I) que arroja en primera instancia la necesidad de implementar urgentemente nuevos instrumentos de planificación en función de las subzonas geográficas del corregimiento de Berlín, como lo es la del Centro Poblado del corregimiento de Berlín. Contrario a lo estipulado por la FAO, los documentos oficiales ignoran temas fundamentales como la diversificación de los cultivos existentes, la búsqueda de proyectos sociales donde se aumente el capital humano y social, proyectos que busquen el equilibrio entre el páramo y el subsistema productivo, donde las externalidades negativas sean mínimas y el territorio se convierta en deseable para inversión privada y pública.

\section{RECOMENDACIONES}

A partir de la visita a campo al Centro Poblado de Berlín y la investigación documental emergen una serie de cuestionamientos que se deben incorporar a la fase de diagnóstico, posterior a la de una cuidadosa lectura del territorio. Solo así se podría consolidar una metodología de intervención contextualizada y acorde con las particularidades del Centro Poblado del corregimiento de Berlín.

En consecuencia, la investigación preliminar arroja una serie de preguntas que dan pie a acciones puntuales por parte de los actuales $y$ futuros planificadores: ise ha concientizado a suficiencia a los habitantes en torno a la fragilidad del suelo del páramo y a la pérdida de ventajas competitivas?, ¿cuál es la posición de la agricultura en el Esquema de Ordenamiento Territorial (EOT) y el Plan de Desarrollo del Municipio?, ise vislumbran posibles proyectos que conduzcan a un certero desarrollo rural sostenible?, iestarían dispuestos los habitantes a capacitarse, cambiar su modus vivendi e inclusive los métodos y tipos de cultivos utilizados históricamente?

Estos cuestionamientos llevan a hilvanar a una serie de acciones primordiales, tanto estatales como sociales para impulsar proyectos agroecológicos, que generen gran impacto en Santander. Una de estas podría ser el conteo, caracterización y tipificación de los predios existentes, pues no se tiene ninguna base estadística actualizada. Asimismo, se requieren estudios minuciosos sobre las externalidades negativas y positivas referidas al actual uso del suelo, así como los que se ocupan de la potencialidad del suelo para otros cultivos, proyectos sociales para la solución de problemáticas asociadas con el agua y proyecciones de agricultura sostenible.

Otro aspecto de vital importancia es el capital social, pues la comunidad necesita un estímulo no solo económico sino de integración como parte de un ecosistema vivo. Las asociaciones son importantes cuando se trata de minifundios ya que se hacen competitivos frente a grandes productores, sin tener desventaja en el cambio de políticas económicas. Es aquí donde técnicas como la realización de una muestra mediante la aplicación de encuestas en cuanto a la percepción de la comunidad sobre las causas de los problemas, los riesgos naturales derivados de estas, y la incorporación de nuevas prácticas agrícolas demuestran su validez. Posteriormente, y concentrándose en los usos del suelo, es necesario trazar los caminos hacia la consolidación de un régimen de baldíos y el establecimiento de políticas estables y duraderas dirigidas a un desarrollo territorial basado en la equidad, o sea, romper 
la brecha de la desigualdad en la tenencia de la tierra y el acceso a recursos económicos de apoyo. Como paso sucesivo, se haría un análisis de contraste entre la problemática actual y lo registrado en el EOT, sumándolo con un inventario físico y cultural de la población, para hacer un diagnóstico sistemático y poder lograr proponer nuevos lineamientos dirigidos a beneficiar las zonas más débiles y a hacer buen uso de las fortalezas territoriales, a fin de incrementar la producción y la calidad de vida de los habitantes.

Finalmente, se llegaría a un punto donde el enfoque sea crear proyectos y estrategias a corto, mediano y largo plazo que estén enlazadas con el Plan de Desarrollo Municipal, en el que incluyan propuestas de equilibrio social y ecológico, a través de una interacción con la comunidad donde se demuestre que es posible llegar a una sostenibilidad social-económica y ambiental con el apoyo del Estado en un marco de transparencia y profesionalismo. En este orden de ideas, es imperioso y más que necesario que la administración pública apunte a un sano proceso de redistribución de la propiedad de la tierra como herramienta para responder a las aspiraciones y exigencias patrimoniales de los campesinos.

Es cierto que estas reformas pueden ser dispendiosas y de un gran nivel de complejidad interdisciplinar, pero también es cierto que hacen parte de los verdaderos cambios que el territorio rural necesita para reducir buena parte de las tensiones sociales que el país afronta. Se habla aquí de una reforma agraria que no solo trate de dividir las grandes propiedades, sino que también cree nuevas formas de gestión, comunidad o grupo. Completamente diferente es la lógica de las reformas agrarias que, en nombre del derecho a la propiedad o la productividad, simplemente divide los predios existentes o coloniza tierras vírgenes.

En conclusión, el camino es largo y por ahora solo queda una certeza: el éxodo rural actual sigue para vaciar el campo. Por tanto, se debe actuar inmediatamente, pues dicho éxodo actúa como una peligrosa válvula de escape a las tensiones rurales existentes que al mismo tiempo transfiere los problemas sociales a las ciudades colombianas.

\section{NOTAS}

I. En la Cumbre Mundial sobre el Desarrollo Sostenible, celebrada en Johannesburgo en 2002, se confirmó la validez del capítulo I4 en materia de ADRS y se renovó el compromiso internacional para cumplir sus objetivos. La ADRS, tal como se concibió originalmente, tenía múltiples aspectos, entre los que destacan la sostenibilidad de las cadenas alimentarias y de los recursos de tierras y aguas, y la idea de la interacción del comercio con los procesos de la ADRS para asegurar unos medios de subsistencia adecuados y garantizar la seguridad alimentaria. "Si bien estas cuestiones siguen siendo válidas -señala el informe- ha cambiado el entorno en que han de afrontarse". Con la aprobación de los Objetivos de Desarrollo para el Milenio de las Naciones Unidas en 2000, el mundo ha adoptado una nueva perspectiva del desarrollo económico y social en un contexto de derechos humanos, en el que la idea central es erradicar la pobreza y el hambre, promover el desarrollo de los recursos humanos, garantizar la sostenibilidad del medio ambiente y establecer una asociación mundial para el desarrollo. Recuperado de http://www.fao.org/Ag/esp/revista/0504sp3.htm

2. La clasificación de fincas se complementa con estudios denominados tipologías de trayectorias, que identifican la evolución histórica de las fincas de una región determinada y las variables o fenómenos que tienen una mayor influencia en esas trayectorias. Esta es una aplicación común de los agrónomos franceses Sebillote, Capillon, Genthon, 
Laurent aunque también hay ejemplos fuera de dicha corriente, concretados por Cornick y Alberti; Suárez y Escobar; Cohan. Velez, L. y Gasto J. (1999) Recuperado en https://repositorio.uc.cl/bitstream/handle/ I I534/8385/0002943 I 5.pdf?sequence = I

3. Julio Iván González Piedra, profesor e investigador de la Universidad de La Habana afirma que en la búsqueda de instrumentos de gestión que posibiliten la transversalidad de políticas sectoriales, diversos países han encontrado en el manejo integral de cuencas un instrumento de planeación y de gestión adecuado. El manejo integral de cuencas no solo permite la gestión equilibrada de los recursos naturales, sino también la integración de los actores involucrados en una sola problemática en lugar de atender varios problemas sectoriales dispersos. Proyección, $N^{\circ}$ I0. El Manejo de Cuencas en Cuba: un instrumento de Planificación Territorial. Recuperado en http:// www.proyeccionrevista.com.ar/revistas/proyeccion-n-10

\section{REFERENCIAS}

Alcaldía del Municipio de Tona. (2016). Plan de Desarrollo Municipal 2016-2019 “Todas y todos construimos un futuro".

Avellaneda, J.A. (1999). Alteración del páramo de Chontales en Boyacá por ganadería y aplicación de plaguicidas en papa.

Corpoica. (1997). Motivaciones y uso de plaguicidas en cultivo de la papa. Corroborado con las entrevistas y encuestas a los agricultores.

DANE. (2015). La cebolla de rama o cebolla junca (Allium fistulosum), una hortaliza de gran importancia en la alimentación humana. Recuperado de http://www.dane.gov.co/files/investigaciones/agropecuario/sipsa/Bol_Insumos_may_20I5.pdf

Departamento de Santander. (2008). Plan Integral de Manejo del Distrito de Manejo Integrado de los Recursos Naturales páramo de Berlín.

Gómez, C. (2009). Agricultura periurbana: nuevos roles de la agricultura de proximidad en la planificación de las periferias urbanas en "Ciudad y Fronteras". Revista M, 6(2). Bucaramanga, Colombia: Ediciones USTA.

Gómez, C. (2007). Agricoltura Periurbana: Una visione dei nuovi ruoli dell'agricoltura per la pianificazione delle periferie urbane. Trabajo de Grado. Doctorado en Pianificazione Urbana, Territoriale e Ambientale. Milano, Italia: Politecnico di Milano.

Gómez, D. (2008). Ordenación Territorial. Madrid, España: Ed. Mundiprensa.

Gómez, D. (2013). Evaluación de Impacto Ambiental. Madrid, España: Ed. Mundiprensa.

Instituto de Investigación de Recursos Biológicos Alexander von Humboldt. (2014). Proyecto páramo Andino. Universidad Nacional de Colombia. Facultad de Ciencias, Departamento de Biología.

Monroy, C. (2009). Caracterización de las prácticas agrícolas asociadas con el uso y manejo 
de plaguicidas en cultivos de papa. Caso vereda Mata de Mora, en el páramo de Merchán, Saboyá (Boyacá). (Trabajo de grado, Maestría en Gestión Ambiental). Pontificia Universidad Javeriana, Facultad de Estudios Ambientales y Rurales. Bogotá, Colombia.

Organización de las Naciones Unidas para la Agricultura y la Alimentación - FAO. (2005). Buenas prácticas agrícolas y ADRS. Recuperado de ftp://ftp.fao.org/docrep/fao/0 I0/al359s/ al359s06.pdf

Organización de las Naciones Unidas - ONU. (s.f.). Objetivos de desarrollo sostenible. 17 objetivos para transformar nuestro mundo. Recuperado el 03/03/2017. Disponible en http:// www.un.org/sustainabledevelopment/es/objetivos-de-desarrollo-sostenible/

Oxfam Internacional. (2016). Desterrado: desigualdades y tierras en América Latina. Bogotá, D.C., Colombia: Oxfam Internacional.

Rodríguez, A., y Meneses, J. (20II). Transformaciones rurales en América Latina y sus relaciones con la población rural en Reunión de Expertos sobre: "Población Territorio y Desarrollo Sostenible", Santiago, Chile: Cepal/Celade.

Vargas, O., Jaimes, V., Castellanos, L., Mora, J. (2004). Proyecto Páramo Andino. Propuesta de actividades de investigación para los páramos de Colombia. Universidad Nacional de Colombia, Instituto de Investigaciones Alexander von Humboldt.

Vélez, L. y Gasto J. (1999). Metodología y determinación de los estilos de arquitectura en escala predia. Recuperado de https://repositorio.uc.cl/bitstream/handle/I I534/8385/0002943 I5. pdf?sequence $=$ I

W. AA. (20I I). Manejo fitosanitario del cultivo de la papa (Solanum tuberosum subsp. andigena y S. phureja). Medidas para la temporada invernal. Bogotá, D.C. Colombia: Produmedios. 